\title{
PRESSURE DEPENDENCE OF THE ACOUSTIC SHEAR MODE IN GaS BY BRILLOUIN SCATTERING
}

\author{
M. Fischer \\ Laboratoire de Dynamique Cristalline et Ultrasons, Departement de Recherches Physiques (U.A. 71), \\ Université Pierre et Marie Curie T 22 E 4, 4 Place Jussieu, F - 75230 Paris-Cédex 05, France \\ and \\ A. Polian, A. Chevy and J.C. Chervin \\ Physique des Milieux tres Condensés (U.A. 782), Université Pierre et Marie Curie T 13 E 4, 4 Place Jussieu, \\ F - 75230 Paris-Cédex 05, France
}

(Received 3 April 1985; in revised form 2 June 1985 by J. Joffrin)

\begin{abstract}
The $C_{44}$ elastic constant of the layer compound $\mathrm{GaS}$ was measured up to $0.6 \mathrm{GPa}$ by Brillouin scattering and its pressure derivative was then compared with linear chain model calculations. The measured value of the pressure derivative is $8 \pm 3$ which is in good agreement with the computed value. A comparison of $C_{44}$ and its pressure derivative with that of other previously measured elastic constants shows a clear separation of the "interlayer" and "intralayer" elastic constants.
\end{abstract}

\section{INTRODUCTION}

GALLIUM SULPHIDE is a hexagonal layer crystal belonging to the $D_{6 h}$ space group. Each layer is made up of two planes of gallium atoms sandwiched between two planes of sulfur atoms. There are two layers in the unit cell ( 8 atoms). Such a structure is characterized by the contrast between the weak Van der Waals type interlayer bonds and the strong ionic-covalent bonds within the layers. As a consequence, the elastic properties of $\mathrm{GaS}$ at room temperature and pressure are strongly anisotropic [1] as well as the pressure derivatives of the elastic constants.

Two methods have been used up to now to measure the variation of elastic moduli vs pressure:

(i) Ultrasonic methods up to $0.7 \mathrm{GPa}$ [2], by which three elastic constants were measured;

(a) $C_{33}$ which is associated with the velocity of the longitudinal phonon propagating along the $c$-axis;

(b) $C_{11}$ and $C_{66}=\left(C_{11}-C_{12}\right) / 2$ which are associated respectively with the velocity of the longitudinal and transverse phonon propagating in the layer planes.

(ii) Brillouin scattering: In a diamond anvil cell [3] up to $17.5 \mathrm{GPa}$, only the longitudinal modes are observ. able, i.e. only $C_{11}$ and $C_{33}$ could be deduced. One important result of this experiment is that the intensity of the restoring forces due to inter- and intralayer bonds became comparable at very high densities.

In neither case could the pressure dependence of $C_{44}$ be measured. This mode is very sensitive to interlayer bonds, and stacking faults could prevent it from propagating. Several samples studied by ultrasonic techniques have shown this anomaly. Brillouin scattering measurements however, where a much smaller volume is required, allow the measurement of this transverse phonon when the scattering volume is suitably selected.

In this paper, we present measurements of the pressure dependence of $C_{44}$ by Brillouin scattering up to $0.6 \mathrm{GPa}$. This result is then discussed and compared with calculations using a linear chain model [4] .

\section{EXPERIMENTAL}

We used a piezoelectrically scanned five pass FabryPerot interferometer without a multiscanning accumulation system. Excitation was produced by $200 \mathrm{~mW}$ of $514.5 \mathrm{~nm}$ radiation from an argon laser containing an intracavity etalon.

The low pressure cell $(\leqslant 0.7 \mathrm{GPa})$ has four access ports, one of which is used for pressurization, another for a sample holder, and the other two optical ports with sapphire windows. We used a $90^{\circ}$ configuration. The scattering geometry was $Z(X Y) X$ where $Z$ is parallel to the $c$-axis. In this configuration, the measured elastic constant combination is $\left(C_{44}+C_{66}\right) / 2$. In order to avoid the depolarization introduced by the sapphire windows, we glued inside the cell a $\lambda / 2$ thin plate on the input window and a polarizer on the output one.

The crystal was grown by the Bridgman technique. It was then cleaved parallel to the layers and cut perpendicular to the layers in order to obtain a $10 \times 7 \times 5$ $\mathrm{mm}^{3}$ parallelepiped. One face was polished perpendicular to the layers. 
The crystallographic quality of the sample was evaluated by measuring the ratio of the backscattered intensity of the Raman forbidden $E_{1 g}\left(\sigma=74 \mathrm{~cm}^{-1}\right.$ at $P=0)$ to that of the Raman allowed $A_{1 \mathrm{~g}}\left(\sigma=188 \mathrm{~cm}^{-1}\right.$ at $P=0$ ). This ratio has been shown to increase rapidly with the density of stacking faults [5]. In the case of our sample, we found a ratio $I\left(E_{1 g}\right) / I\left(A_{1 g}\right)=0.02$, which indicate a good quality crystal. Nevertheless, even with that quality, $C_{44}$ could not be measured by ultrasonics in this sample after the first pressure run in which $C_{33}$ was measured.

\section{RESULTS AND DISCUSSION}

\section{(a). Results}

The free spectral range of the interferometer was $1.9947 \pm 0.0004 \mathrm{~cm}^{-1}$. The pressure dependence of the ordinary refractive index is [3]:

$n_{0}(P)=2.73+0.05 P$,

where $P$ in GPa is the hydrostatic pressure. In the geometry of the experiment, the Brillouin frequency shift in $\mathrm{cm}^{-1}$ is related to the sound velocity by:

$\Delta \sigma=\frac{n V \sqrt{2}}{\lambda c}$,

with $n=n_{0}(P)$, the ordinary refractive index, $V$ the sound velocity, $\lambda$ the wavelength of the incident laser light and $c$ the velocity of light. The sound velocity is in turn related to the elastic constants by:

$V=\frac{C_{44}+C_{66}}{2 \rho}$,

where $C_{66}=\left(C_{11}-C_{12}\right) / 2$ and $\rho$ is the density which at zero pressure is $3.913 \mathrm{~g} / \mathrm{cm}^{3}$.

Figure 1 shows a typical Brillouin spectrum. In that geometry, the peaks are rather weak. In Fig. 2 is shown the pressure dependence of the sound velocity deduced using equation (2). The pressure dependence of $C_{44}$ is then deduced using the values of $C_{66}$ obtained from ultrasonics results [4] and the density deduced from X-ray measurements of the $a$ and $c$ parameters [6] .

Due to the weak Brillouin signal, the error in $V$ is of the order of $3 \%$; in the density, it is of the order of $1.5 \%$. Consequently the pressure derivative of $C_{44}$ could only be obtained with a precision of $40 \%$. We obtained:

$\frac{\mathrm{d} C_{44}}{\mathrm{~d} P}=8 \pm 3$.

\section{(b). Discussion}

The experimental value of $\left(\mathrm{d} C_{44} / \mathrm{d} P\right)$ can be compared with the value obtained from the linear chain model described in [4] .

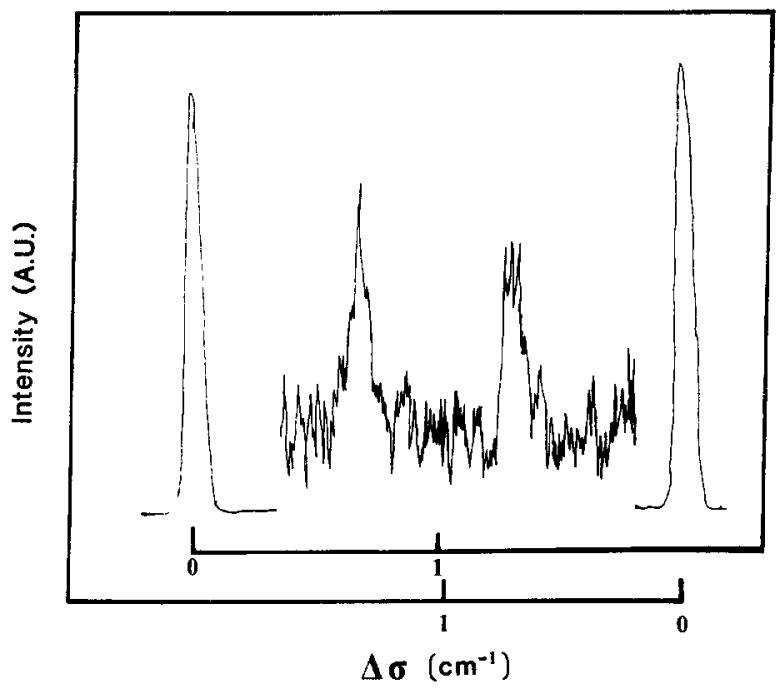

Fig. 1. Brillouin spectrum of $\mathrm{GaS}$ at $0.461 \mathrm{GPa}$ in the $Z(X Y) X$ scattering geometry. Exciting light is the $514.5 \mathrm{~nm}$ line of an argon laser with a power of $200 \mathrm{~mW}$. F.S.A. $=1.9947 \mathrm{~cm}^{-1}$.

In that model, there are interactions between all pairs of atoms inside a layer, and only between the sulfur atoms of adjacent layers for the interlayer bonds. Due to the crystal symmetry, it is sufficient to introduce 10 independent parameters $\gamma_{\alpha \beta}$, which are summations of microscopic coupling parameters. The crystal can be considered as a stacking along the six-fold axis $c$ of monatomic planes of equivalent atoms with the sequence ...S $-\mathrm{Ga}-\mathrm{Ga}-\mathrm{S} \ldots \mathrm{S}-\mathrm{Ga}-\mathrm{Ga}-\mathrm{S} \ldots$ where the intralayer bonds are in full lines and the interlayer one in dotted lines. Then, for an elastic wave propagating along the $c$-axis, the crystal is equivalent to a linear chain. The models of Wieting [7] and Maiti [8] are particular cases of this model.

In this model, which ignores the Coulomb interaction, the coefficient of the dynamical matrix for $k \| c$ can easily be calculated if one introduces the parameter

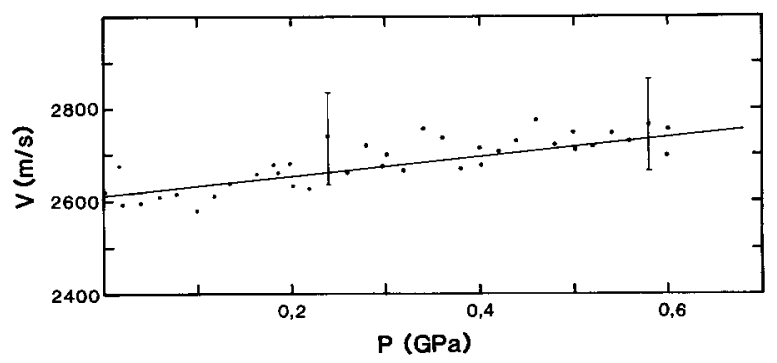

Fig. 2. Sound velocity of the measured transverse phonon vs pressure. Error bars are shown for some points. 
Table 1. Measured elastic constants in $10^{10} \mathrm{~Pa}$ and their pressure derivatives and logarithmic pressure derivatives in $10^{-10} \mathrm{~Pa}^{-1}$ at room pressure

\begin{tabular}{lllll}
\hline & $C_{11}$ & $C_{33}$ & $C_{44}$ & $C_{66}$ \\
\hline$C_{i j}(P=0)$ & $12.33 \pm 0.07$ & $3.85 \pm 0.04$ & $0.996 \pm 0.015$ & $4.43 \pm 0.17$ \\
$\mathrm{~d} C_{i j} / \mathrm{d} P$ & $9.8 \pm 1.2$ & $22.7 \pm 1.3$ & $6 \pm 2$ & $2.4 \pm 0.3$ \\
$\mathrm{~d} \log C_{i j} / \mathrm{d} P$ & 0.8 & 5.9 & 6.0 & 0.5 \\
\hline
\end{tabular}

$\gamma_{\alpha \beta}=\sum_{\nu} \Phi_{X X X}\left(\begin{array}{ll}0 & \nu \\ \alpha & \beta\end{array}\right)$,

where $\Phi$ is the second order coupling parameter between the atoms $\left(\begin{array}{l}0 \\ \alpha\end{array}\right)$ and $\left(\begin{array}{l}\mu \\ \beta\end{array}\right)$. A relation exists between the pressure derivative of $C_{44}$ and that of $\gamma_{14}$ which is defined as $\psi_{14}$ for this transverse mode [4]:

$\frac{\mathrm{d} \ln \Psi_{14}}{\mathrm{~d} P}=\left(1-\frac{\Psi_{14}}{\Gamma_{\perp}}\right)\left(\frac{\mathrm{d} \ln C_{44}}{\mathrm{~d} P}-2 B_{\perp}+B_{\|}\right)$,

where $\Gamma_{\perp}$ is the shear stiffness of an isolated layer, $B_{\|}$ and $B_{\perp}$ are the linear compressibilities respectively parallel and perpendicular to the $c$-axis. $\mathrm{d} \log \psi_{14} / \mathrm{d} P$ can also be related to the principal frequencies $\sigma_{1}\left(E_{1 u}^{(2)}\right)$, $\sigma_{4}\left(E_{2 g}^{(1)}\right), \sigma_{4}\left(E_{2 g}^{(2)}\right)$ and their pressure derivatives by:

$\frac{\mathrm{d} \ln \Psi_{14}}{\mathrm{~d} P}=\frac{1}{1+\alpha}\left(\frac{\mathrm{d} \ln \sigma_{4}^{2}}{\mathrm{~d} P}+\alpha \frac{\mathrm{d} \ln \sigma_{4}^{\prime 2}}{\mathrm{~d} P}\right)$,

with

$\alpha=\frac{\sigma^{2}-S^{2}}{\sigma_{4}^{\prime 2}-S^{2}}$,

and

$S^{2}=\frac{\left(1+m_{2} / m_{1}\right) \sigma_{4}^{2}\left(\sigma_{1}^{2}-\sigma_{4}^{2}\right)}{\sigma_{1}^{2}-\left(1+m_{2} / m_{1}\right) \sigma_{4}^{2}}$,

where $m_{1}$ and $m_{2}$ are the atomic masses of sulfur and gallium atoms.

Using the experimental values for the frequencies and their pressure derivatives [5] we obtain $d \log \psi_{14}$ / $\mathrm{d} P=4.45 \pm 1.2$.

Using equation 5 we obtain:

$\mathrm{d} C_{44} / \mathrm{d} P=4.1 \pm 1.3$.

Due to the large uncertainties the experimental and the calculated values are consistent.
From Table 1 which gives the pressure derivatives of four of the elastic moduli and their logarithms, one can see that the elastic constants are separated in two well defined groups: Those for which $\mathrm{d} \log C_{i j} / \mathrm{d} P$ is of the order of 6 or more $\left(C_{33}\right.$ and $\left.C_{44}\right)$ and those for which it is smaller than $1\left(C_{11}\right.$ and $\left.C_{66}\right)$. This is due to the fact that the pressure variation of $C_{33}$ and $C_{44}$ is related to the weak interlayer forces which varies rapidly with pressure, whereas $C_{11}$ and $C_{66}$ are "intralayer" elastic moduli and vary much more slowly (one order of magnitude).

\section{CONCLUSION}

Using Brillouin scattering, we have measured the variation of the elastic constant $C_{44}$ with pressure. There is good agreement between the experimental values and values calculated with a linear chain model. Comparison with published measurements on $C_{11}, C_{33}$ and $C_{66}$ permit the separation of the elastic constants into two groups. On the one hand the "interlayer" constants $C_{33}$ and $C_{44}$ for which $\mathrm{d} \log C_{i j} / \mathrm{d} P$ is of the order of 6 , and the "intralayer" moduli $C_{11}$ and $C_{66}$ on the other hand for which $\mathrm{d} \log C_{i j} / \mathrm{d} P$ is less than 1 .

\section{REFERENCES}

1. A. Polian, M. Grimsditch, M. Fischer \& M. Gatulle, J. Phys. Lett. 43, L405 (1982).

2. M. Gatulle, M. Fischer \& A. Chevy, Phys. Status Solidi (b) 119, 327 (1983).

3. A. Polian, J.M. Besson, M. Grimsditch \& H. Vogt, Phys. Rev. B25, 2767 (1982).

4. M. Gatulle \& M. Fischer, Phys. Status Solidi (b) 121, 59 (1984).

5. A. Polian, J.M. Besson \& J.C. Chervin, Phys. Rev. B22, 3049 (1980).

6. H. D'Amour \& W.B. Holzapfel, to be published.

7. T.J. Wieting, Solid State Commun. 12, 931 (1973).

8. C.A. Maiti \& P.N. Ghosh, Solid State Commun. 42, 149 (1982). 